\title{
Algumas Simulações de Efeitos de Mobilidade de Renda Sobre o Nível de Bem-Estar
}

\author{
Erik Alencar de Figueirêdo*, Flávio Augusto Ziegelmann ${ }^{\dagger}$
}

\author{
Conteúdo: 1. Introdução; 2. Resultados; 3. Considerações Finais; A. Apêndice; \\ Palavras-chave: Mobilidade de Renda; Bem-Estar Econômico. \\ Códigos JEL: $\quad$ I30; 139.
}

Este estudo busca mensurar o impacto da mobilidade de renda sobre o nível de bem-estar econômico no Brasil. Para tanto, utiliza-se o modelo de consumo intertemporal proposto por Gottschalk e Spolaore (2002). Tal suporte teórico permite a avaliação isolada dos efeitos das duas fontes de mobilidade: a reversibilidade e a independência da origem. As simulações realizadas sugeriram que a flexibilização da estrutura social brasileira, representada pelo aumento na mobilidade de renda, gera um impacto positivo sobre o padrão de bem-estar econômico do país.

This study intends to measure the relationship between Brazilian economic welfare and income mobility. To achieve that it was used a model of intertemporal consumption proposed by Gottschalk e Spolaore (2002). This theoretical support allows to evaluate isolated the two sources of mobility: the reversibility and the source independence. The simulations suggest that flexibilizantion of Brazilian social structure, represented by an increase of income mobility, results a positive impact on the economic welfare.

\section{INTRODUÇÃO}

Este estudo mensura o efeito da mobilidade de renda sobre o nível de bem-estar econômico no Brasil. Para tanto, considerou-se o modelo de consumo intertemporal desenvolvido por Gottschalk e Spolaore (2002). A utilização deste ferramental tornou possível a separação dos efeitos das diferentes fontes teóricas da mobilidade, ou seja, da reversibilidade e da independência da origem. A reversibilidade está associada ao grau em que as posições econômicas são revertidas ao longo do tempo, ou seja, a capacidade dos pobres tornarem-se ricos e os ricos tornarem-se pobres. Já a independência da origem diz respeito ao quanto a posição econômica atual depende da posição econômica no passado.

\footnotetext{
* Departamento de Economia, Universidade Federal da Paraiba (UFPB). E-mail: eaf igueiredo@gmail . com

${ }^{\dagger}$ Departamento de Estatística, Universidade Federal do Rio Grande do Sul (UFRGS).E-mail: flavioz@ufrgs .br
} 
Foram construídas três funções de bem-estar social representando as sociedades estática (sem mobilidade), reversível e independente da origem. Levaram-se em conta as características relativas às aversões sociais à desigualdade, às flutuações intertemporais no consumo e ao risco. A diferença entre os índices de Atkinson estendidos, calculados para as três sociedades, deram uma ideia sobre a desejabilidade da mobilidade de renda.

Em resumo, as simulações demonstraram que a mobilidade de renda gera um impacto positivo sobre o nível de bem-estar econômico. A comparação dos resultados brasileiros com os internacionais, reforçaram a hipótese da influência do nível da desigualdade sobre a magnitude dos incrementos do bem-estar associados a mobilidade de renda. Ou seja, nações que apresentam grandes disparidades de renda e significativas parcelas da população "localizadas" nos estratos inferiores da distribuição, tendem a se beneficiar mais com a mobilidade.

A seguir, faz-se a apresentação dos resultados do estudo, evidenciando os detalhes relacionados ao banco de dados, processo de simulação e a discussão dos resultados. A seção três é reservada para as considerações finais. Uma síntese do modelo teórico e a apresentação do índice de Atkinson estendido, podem ser encontradas no Apêndice A.

\section{RESULTADOS}

Os resultados dessa seção baseiam-se na estrutura teórica desenvolvida por Gottschalk e Spolaore (2002). ${ }^{1}$ Em resumo, o exercício consiste em calibrar funções de bem-estar considerando três sociedades: estática, reversível e independente da origem. Em seguida, calculam-se os índices de Atkinson estendidos, atribuindo valores $a d$ hoc para os níveis de aversões sociais à desigualdade, às flutuações intertemporais no consumo e ao risco, representadas, respectivamente, por $\epsilon, \rho$ e $\gamma$. A diferença entre os índices de Atkinson estendidos, calculados para as três sociedades, representam os ganhos de bem-estar resultantes da mobilidade de renda.

\subsection{Dados}

Este tópico tem como objetivo discutir a natureza e a construção do banco de dados. Optou-se pela variável "renda pessoal"2 oriunda da Pesquisa Nacional de Amostras por Domicílios (PNAD), realizada pelo Instituto Brasileiro de Geografia e Estatística (IBGE), tendo como base o mês de setembro dos respectivos anos. O primeiro passo foi o da conversão das moedas e o deflacionamento. ${ }^{3}$ Para tanto, utilizou-se o procedimento sugerido por Corseuil e Foguel (2002).

Foram excluídas as rendas negativas e iguais a zero, pois o cálculo do índice de Atkinson necessita que o vetor de dados seja estritamente positivo. Outra característica relacionada a inferência deste indicador, diz respeito à sua sensibilidade a valores muito próximos a zero, principalmente quando o parâmetro de aversão à desigualdade situa-se no intervalo $1 \leq \epsilon \leq 3$. O fato é que mesmo um pequeno número de observações com esta característica, possui forte influência sobre o índice. Por conta disso, as rendas quase-zero foram excluídas da amostra. 0 método de exclusão baseou-se na estatística proposta por Cowell e Vitoria-Feser (1996), ou seja:

\footnotetext{
1É importante destacar que o modelo teórico não possui uma teoria de incentivos para a produção dos níveis de consumo e renda. Este tópico constitui um importante desafio para a literatura especializada. Para detalhes, ver Fields e Ok (2001).

${ }^{2} \mathrm{~A}$ utilização da renda é uma prática comum nos estudos de mobilidade internacionais, dada a limitação referente à disponibilidade de dados de consumo. Contudo, esta prática postula a existência de imperfeições no mercado de crédito. Neste caso, os agentes econômicos se veem impossibilitados de tomar empréstimos. Na verdade, esta suposição envolve um importante tópico da literatura empírica, qual seja: considerar a renda ou o consumo como indicador de bem-estar? Argumenta-se que a trajetória do consumo pode ser mais suave ao longo do ciclo da vida, enquanto a renda está sujeita a variações sazonais. Para detalhes, ver o capitulo 2 de Duclos e Araar (2006).

${ }^{3}$ Todos os valores estão expressos em Reais de janeiro de 2005.
} 


$$
I F(x, y)=\frac{x^{\alpha}+\sum_{i=1}^{n} w_{i} \frac{y_{i}^{\alpha}}{n}\left(\alpha-1-\frac{\alpha x}{\mu(y)}\right)}{\left(\alpha^{2}-\alpha\right) \mu(y)^{\alpha}}
$$

Onde $I F$ representa a função de influência dos dados sobre a medida de Atkinson. A variável $y$ representa o vetor de renda, $w$ o peso da observação, $x$ a renda mais baixa observada na distribuição e $\alpha=1-\epsilon$. Os valores de $x$ associados a uma $I F$ superior a $10 \%$ foram excluídos. Em média, esta exclusão não ultrapassou o percentual de $0,007 \%$ da amostra em cada $\epsilon$ considerado.

\subsection{Simulações}

Nesta seção, serão apresentados e discutidos os principais resultados do estudo. As simulações foram efetuadas com base em dados relativos à renda brasileira em dois pontos no tempo: os anos de 1987 e 2005. Calculam-se funções de bem-estar para as sociedades estática, reversível e independente da origem. No caso da sociedade estática, o nível de renda do período 1 (1987) é mantido no segundo. A simulação relativa a reversibilidade, considera uma inversão no padrão distributivo referente ao primeiro período. Logo, nestes dois primeiros casos, só foi utilizado o vetor de dados relacionado ao ano de 1987. Por fim, a consideração da independência da origem introduz a incerteza quanto ao consumo no período 2, ou seja, o ano de 2005. Neste caso, o primeiro passo é obter o equivalente certeza a partir da fórmula (A.4). Em seguida, gerou-se uma expectativa de renda futura, baseada na renda de 1987 e no equivalente certeza. Esta inferência é obtida a partir da estimação da densidade conjunta, utilizando-se da técnica do kernel smoothing. ${ }^{4}$

A Tabela 1 apresenta duas informações. Primeiro, observam-se as estimativas para os índices de Atkinson estendidos. Estes indicadores mensuram a perda de bem-estar resultante da desigualdade e da imobilidade de renda. Logo, valores próximos a 1 (um) sugerem à existência de um baixo padrão de bem-estar econômico. A segunda informação está relacionada aos ganhos de bem-estar associados às diferentes fontes de mobilidade. A análise é procedida a partir da simulação de três situações sumarizadas nas colunas 1, 2 e 3. A diferença entre as simulações reside na atribuição de valores diferenciados para os parâmetros $\epsilon, \rho$ e $\gamma^{5}$ Na primeira coluna, assume-se a existência da preferência pela igualdade $(\epsilon=4)$ e as inexistências de flutuações intertemporais e do risco relativo ao nível de consumo no segundo período. $\mathrm{O}$ valor de $\epsilon$ é mantido nas demais colunas, variando apenas os parâmetros $\rho$ e $\gamma$. Na coluna 2, considera-se $\rho=2$ e $\gamma=0$, ou seja, incorpora-se as flutuações intertemporais ao modelo. Na terceira coluna, todos os parâmetros assumem valores diferentes de zero. Neste caso, o $\gamma=2$ implica na existência do risco relativo ao consumo no período seguinte.

Na coluna 1, observa-se um $A_{s}=0,9444$, ou seja, em uma sociedade estática, com um parâmetro de aversão à desigualdade igual a 4, a perda de bem-estar é elevada. Neste sentido, a mobilidade de renda gera ganhos coletivos. Note que os índices relativos a reversibilidade $\left(A_{r}\right)$ e à independência da origem $\left(A_{0}\right)$ são inferiores ao $A_{s}$. De fato, a reversibilidade propicia um ganho de bem-estar na ordem de $0,0206\left(A_{s}-A_{r}\right)$. Já a independência da origem é responsável por uma elevação em torno de $0,0144\left(A_{r}-A_{0}\right)$. Consequentemente, a mobilidade total, ou seja, a soma das duas fontes, gera um incremento de 0,0351 .

Os resultados da coluna 2 apresentam um movimento similar aos da coluna 1, variando apenas os valores absolutos. Logo, pode-se afirmar que a mobilidade gera um aumento no bem-estar. Como já ressaltado, nesta simulação não considera-se o risco relativo ao consumo no período 2 ( $\epsilon=4, \rho=2$ e $\gamma=0)$. A perda de bem-estar associada a uma sociedade estática é representada por $A_{s}=0,7774$. Há uma redução deste valor quando introduz-se a mobilidade. O ganho total de bem-estar é de 0,1402, sendo 0,0825 da reversibilidade e 0,0577 da independência da origem.

\footnotetext{
${ }^{4}$ A seleção do parâmetro de alisamento deu-se a partir de método plug-in simples. Para detalhes, ver Härdle (1990).

${ }^{5}$ Os valores foram selecionados de modo a satisfazer os requisitos da Proposição 1 de Gottschalk e Spolaore (2002).
} 
A simulação da coluna 3 incorpora a aversão ao risco referente ao consumo no período seguinte. Em outras palavras, a expectativa de consumo relativa ao período 2 (no caso deste estudo, o ano de 2005), que é formulada no período 1 (no ano de 1987), deve considerar um fator de risco $\gamma=2$. Esperase, com isso, mensurar os ganhos de bem-estar associados a existência do "véu da ignorância", isto é, referente à incerteza quanto ao consumo futuro. Note que os valores para $A_{s}$ e $A_{r}$ são idênticos aos observados na coluna 2 , o que é natural, dado que estes indicadores são calculados tendo como base apenas os dados de 1987. Entretanto, $A_{0}$ apresenta o menor valor entre todos os índices já estimados, contribuindo para o elevado ganho total de bem-estar $(0,1458)$. Vale ressaltar que os índices e os ganhos de bem-estar são os mais expressivos dentre todas as simulações realizadas.

Tabela 1: Impacto da mobilidade sobre o bem-estar econômico

\begin{tabular}{|c|c|c|c|}
\hline Parâmetros & $(1)$ & $(2)$ & (3) \\
\hline$\epsilon$ & 4 & 4 & 4 \\
$\gamma$ & 0 & 2 & 2 \\
\multicolumn{3}{|c|}{ Resultados } \\
\hline$A_{s}$ & 0,9444 & 0 & 2 \\
$A_{r}$ & 0,9237 & 0,69479 & 0,7774 \\
$A_{0}$ & 0,9093 & 0,6372 & 0,6316 \\
Independência da origem $\left(A_{r}-A_{0}\right)$ & $-0,0206$ & $-0,0825$ & $-0,0825$ \\
\hline Reversibilidade $\left(A_{s}-A_{r}\right)$ & $-0,0577$ & $-0,0633$ \\
\hline Total $\left(A_{s}-A_{0}\right)$ & $-0,0351$ & $-0,1402$ & $-0,1458$ \\
\hline
\end{tabular}

Fonte: Dados da pesquisa.

A observação dos resultados sugere os seguintes destaques:

a) dado o alto grau de desigualdade observado no Brasil, as simulações relativas à sociedade estática mostraram índices $\left(A_{s}\right)$ sempre superiores às das demais situações. Sendo assim, a mobilidade de renda constituiu uma importante fonte para a melhoria do bem-estar;

b) neste contexto, destacam-se os ganhos referentes à reversibilidade e;

c) o comportamento dos valores calculados para independência da origem. Este último tópico merece um maior destaque. Note que há um crescimento do valor, em termos absolutos, quando se transita da primeira para a terceira coluna, ou seja, a desigualdade de renda, caracterizada pela forte concentração de indivíduos na parte inferior da distribuição, faz com que torne-se preferível uma situação onde a renda presente, no ponto de vista intrageracional, ou a renda dos pais, na visão intergeracional, não interfira na renda futura.

A comparação do resultado brasileiro com os internacionais propiciará um melhor entendimento para o fenômeno investigado. Neste sentido, destaca-se o estudo de Gottschalk e Spolaore (2002), que investiga os casos americano e alemão. Dado que as metodologias são idênticas, será possível uma comparação direta entre as realidades dos três países. Seus resultados são sumarizados nas Tabelas $2 \mathrm{e}$ 3.

Em primeiro lugar, destacam-se os comportamentos dos índices de Atkinson estendidos relativos à sociedade estática. No caso norte-americano, ele estabiliza-se em torno de 0,6660. Os índices alemães são menores (girando em torno de 0,4060 ). Logo, ambos são inferiores aos brasileiros, o que era 
Tabela 2: Impacto da mobilidade sobre o bem-estar econômico nos EUA

\begin{tabular}{|c|c|c|c|}
\hline Parâmetros & $(1)$ & $(2)$ & $(3)$ \\
\hline$\epsilon$ & 4 & 4 & 4 \\
$\gamma$ & 0 & 2 & 2 \\
$\gamma$ & 0 & 0 & 2 \\
\hline \multicolumn{3}{|c|}{ Resultados } \\
$A_{s}$ & 0,6660 & 0,6680 & 0,6680 \\
$A_{0}$ & 0,5650 & 0,6220 & 0,6220 \\
Reversibilidade $\left(A_{s}-A_{r}\right)$ & 0,3540 & 0,5090 & 0,5780 \\
Independência da origem $\left(A_{r}-A_{0}\right)$ & $-0,1010$ & $-0,0460$ & $-0,0460$ \\
\hline Total $\left(A_{s}-A_{0}\right)$ & $-0,3120$ & $-0,1140$ & $-0,0440$ \\
\hline
\end{tabular}

Fonte: Gottschalk e Spolaore (2002).

esperado dado que os EUA e a Alemanha apresentam uma menor desigualdade de renda. ${ }^{6}$ Sob este panorama, a reversibilidade mostra-se mais relevante, como fonte de incremento de bem-estar, para 0 caso brasileiro (em média 0,0300 superior aos valores dos EUA e Alemanha).

Tabela 3: Impacto da mobilidade sobre o bem-estar econômico na Alemanha

\begin{tabular}{|c|c|c|c|}
\hline Parâmetros & $(1)$ & $(2)$ & $(3)$ \\
\hline$\epsilon$ & 4 & 4 & 4 \\
$\gamma$ & 0 & 2 & 2 \\
& 0 & 0 & 2 \\
\hline \multicolumn{3}{|c|}{ Resultados } \\
$A_{s}$ & 0,4010 & 0,4060 & 0,4060 \\
$A_{r}$ & 0,2840 & 0,3510 & 0,3510 \\
Reversibilidade $\left(A_{s}-A_{r}\right)$ & 0,1690 & 0,2500 & 0,3100 \\
Independência da origem $\left(A_{r}-A_{0}\right)$ & $-0,1170$ & $-0,0550$ & $-0,0550$ \\
\hline Total $\left(A_{s}-A_{0}\right)$ & $-0,2320$ & $-0,1010$ & $-0,0410$ \\
\hline
\end{tabular}

Fonte: Gottschalk e Spolaore (2002).

Nos casos americano e alemão, os ganhos gerados pela independência da origem são decrescentes, ao contrário do que observa-se no Brasil. Este comportamento sugere que a incerteza quanto ao consumo futuro são mais significativos para uma nação desigual como o Brasil do que para sociedades com níveis de distribuição de renda mais igualitários. A comparação entre os resultados dos EUA com os da Alemanha reforça esta conclusão. Dado que os EUA apresentam uma distribuição de renda

\footnotetext{
${ }^{6}$ Ver United Nations Development Program (2006).
} 
mais desigual, seus ganhos associados à independência da origem são maiores do que os observados na Alemanha.

No entanto, este resultado constitui um paradoxo, pois segundo Alesina et alii (2001) a desigualdade de renda possui um efeito negativo sobre a "felicidade" dos europeus mas não sobre a "felicidade" da população norte-americana, pois os americanos associam pobreza a ineficiência, enquanto que os europeus a consideram "falta de sorte". Neste sentido, a independência da origem, que reduz a desigualdade nos múltiplos períodos, deveria ser mais relevante para a Alemanha do que para os EUA. Uma das possíveis explicações para este comportamento reside na percepção da mobilidade social e de sua associação com a independência da origem. Para Gottschalk e Spolaore (2002) ela seria mais forte entre os norte-americanos. Por fim, o que pode-se afirmar é que estes resultados destacama heterogeneidade das sociedades. Ou seja, cada nação possui o seu próprio ponto de ótimo para a estrutura da mobilidade.

Para o caso do Brasil, esta questão fica em aberto. O que se pode afirmar é que o país apresenta uma elevada rigidez em sua estrutura social. Esta afirmação é corroborada, entre outros, por Ferreira e Veloso (2006) que, utilizando os dados da PNAD referentes ao ano de 1996, detectam uma baixa mobilidade intergeracional no Brasil. Isto é, os salários dos pais tendem a ser "transferidos" para os filhos em uma magnitude superior ao observado em países desenvolvidos.

Neste contexto, o aumento da mobilidade de renda passa a representar uma "quebra" nesta estrutura, gerando, com isso, uma melhor distribuição dos rendimentos e, por conseguinte, uma situação caracterizada pelo maior nível de bem-estar econômico. Entretanto, este resultado é sustentado pela hipótese da ausência de perdas de bem-estar ex-ante, associadas ao aumento da incerteza oriunda da mobilidade (mais epecificamente, decorrente da independência da origem). Sendo assim, uma sugestão futura para a pesquisa será abordar os impactos ex-ante e ex-post. Desta forma, o incremento de bem-estar só ocorreria se o segundo efeito dominasse o primeiro.

\section{CONSIDERAÇÕES FINAIS}

Este estudo mensurou o efeito da mobilidade de renda sobre o nível de bem-estar econômico no Brasil. Para tanto, foram realizadas três simulações, supondo à existência das sociedades estática, reversível e independente da origem. Considerou-se que a aversão à desigualdade domina as aversões à flutuação intertemporal no consumo e ao risco futuro $(\epsilon>\rho$ e $\epsilon>\gamma)$.

Os resultados demonstraram que a mobilidade de renda gera um impacto positivo sobre o nível de bem-estar econômico brasileiro. Neste contexto, os valores relativos a reversibilidade são coerentes, dado o alto grau de desigualdade de renda observado no Brasil. Isto é, como a distribuição de renda brasileira é caracterizada pela grande concentração de pessoas na parte inferior da densidade, uma reversão desta realidade, com os pobres tornando-se ricos e os ricos tornando-se pobres, geraria um maior bem-estar social.

Este mesmo argumento pode ser empregado para explicar o comportamento dos ganhos relacionados a independência da origem. Dada uma situação inicial desfavorável no tempo $t$, é preferível que ela não interfira na posição do indivíduo em $t+1$. Sendo assim, o "véu da ignorância" propicia ganhos do ponto de vista agregado.

A comparação dos resultados brasileiros com os internacionais apontaram para a influência do nível da desigualdade sobre a magnitude dos incrementos do bem-estar associados a mobilidade de renda. Ou seja, nações que apresentam grandes disparidades de renda e significativas parcelas da população "localizadas" nos estratos inferiores da distribuição, tendem a se beneficiar mais com a mobilidade.

Por fim, os resultados sugerem que uma flexibilização na estrutura social brasileira, representada pelo o aumento na mobilidade de renda, contribuirá para a melhoria na distribuição dos rendimentos e, consequentemente, gerará um acréscimo no nível de bem-estar econômico. Entretanto, convém 
destacar que a estrutura teórica aqui utilizada não admite perdas a priori relacionadas ao aumento na incerteza na economia. Sendo assim, a inclusão desta possibilidade norteará uma pesquisa futura.

\section{BIBLIOGRAFIA}

Alesina, A., Di Tella, R., \& MacCulloch, R. (2001). Inequality and hapiness: Are Europeans and Ameircans different? Technical Report W8198, NBER.

Atkinson, A. B. (1970). On the measurement of inequality. Journal of Economic Theory, 2:244-263.

Atkinson, A. B. \& Bourguignon, F. (1982). The comparison of multidimensional distributions of economic status. Review of Economic Studies, 49:183-201.

Corseuil, C. \& Foguel, M. (2002). Uma sugestão de deflatores para rendas obtidas a partir de algumas pesquisas domiciliares do IBGE. Mercado de Trabalho-Conjuntura e Análise, 1:57-63.

Cowell, F. A. \& Vitoria-Feser, M.-P. (1996). Robustness properties of inequality measures. Econometrica, 64:77-101.

Duclos, J.Y. \& Araar, A. (2006). Poverty and Equity: Measurement, Policy and Estimation with DAD. Springer, New York.

Ferreira, S. G. \& Veloso, F. A. (2006). Intergeneracional mobility of wages in Brazil. Brazilian Review of Econometrics, 26:181-211.

Fields, G. S. \& Ok, E. A. (2001). The measurement of income mobility: An introduction to the literature. In Silber, J., editor, Handbook on Income Inequality Measurement. Kluwer Academic Press, Boston.

Gottschalk, P. \& Spolaore, E. (2002). On the evaluation of economic mobility. Review of Economic Studies, 69:191-208.

Härdle, W. (1990). Applied Nonparametric Regression. Cambridge University Press, New York.

Harsanyi, J. (1955). Cardinal welfare, individualistic ethics and interpersonal comparisons of utility. Journal of Political Economy, 63:309-319.

Kreps, D. \& Porteus, E. (1978). Temporal resolution of uncertainty and dynamic choice theory. Econometrica, 46:185-200.

Rawls, J. (1970). A Theory of Justice. Oxford University Press, Oxford.

United Nations Development Program (2006). Human development report.

von Neumann, J. \& Morgenstern, O. (1944). Theory of Games and Economic Behavior. Princeton University Press, Princeton. 


\section{A. APÊNDICE}

\section{DISTRIBUIÇÃO DE RENDA E BEM-ESTAR ECONÔMICO}

Este apêndice apresentará uma versão sintética do modelo teórico utilizado no estudo. Maiores detalhes podem ser obtidos Gottschalk e Spolaore (2002).

\section{A.1. Reversibilidade e independência da origem}

Considere uma sociedade na qual os indivíduos vivem dois períodos. Em cada período, metade da população apresenta um consumo baixo, $c^{B}>0$, e a restante um consumo alto, $c^{A}>c^{B}>0$. Define-se $\pi\left(c^{i}, c^{j}\right)$ como a probabilidade dos indivíduos consumirem $c^{j}$ no segundo período, dado que eles consumiram $c^{i}$ no primeiro período ( $\operatorname{com} i, j=A, B$ ). Suponha ainda que uma proporção dos agentes, representada por $(1-\delta)$, possui o mesmo nível de consumo em ambos os períodos e a outra, representada por $(\delta)$, apresenta consumos diferentes nos dois períodos. Sendo assim, essa sociedade pode ser representada por:

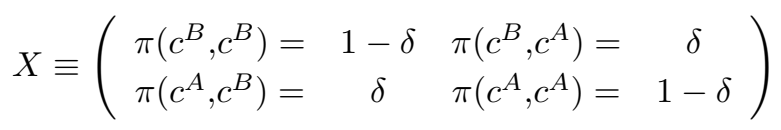

Uma sociedade é dita estática, se $\delta=0$; caso $\delta \neq 0$, ela possuirá mobilidade resultante:

a) da independência da origem, se $\delta=1 / 2$, ou seja, o consumo no segundo período é completamente independente do consumo observado no primeiro período ou;

b) da completa reversibilidade, se $\delta=1$, neste caso, todos os ricos tornar-se-ão pobres e os pobres, por sua vez, tornar-se-ão ricos.

Isso posto, a associação entre o nível de mobilidade dessa sociedade e seu respectivo bem-estar, pode ser estabelecida a partir da seguinte função de bem-estar social:

$$
W=\sum_{i} \sum_{j} G\left[u\left(c^{i}\right)+v\left(c^{j}\right)\right] \pi\left(c^{i}, c^{j}\right)
$$

onde $G$ representa uma transformação côncava ${ }^{7}, u(\cdot)$ e $v(\cdot)$ são funções de utilidade côncavas e $\pi\left(c^{i}, c^{j}\right)$ designa a função de densidade conjunta, estimada a partir dos vetores de consumo. Vejamos um exemplo, suponha que a função de utilidade para dois períodos seja dada por:

$$
\begin{aligned}
W & =\frac{1}{2}\left\{(1-\delta) G\left[u\left(c^{B}\right)+v\left(c^{B}\right)\right]+(1-\delta) G\left[u\left(c^{A}\right)+v\left(c^{A}\right)\right]\right. \\
& \left.+\delta G\left[u\left(c^{A}\right)+v\left(c^{B}\right)\right]+\delta G\left[u\left(c^{B}\right)+v\left(c^{A}\right)\right]\right\}
\end{aligned}
$$

Assim, se $G$ é côncava, $d W / d \delta>0$. Ou seja, quanto maior o $\delta$, maior o bem-estar. Grosso modo, este resultado é coerente com diversas "teorias da justiça" que consideram o aumento da mobilidade como fonte de bem-estar.

Neste contexto, Atkinson e Bourguignon (1982) apontam uma conclusão importante: quando têm-se funções do bem-estar do tipo (A.1), ou seja, $W=\sum_{i} \sum_{j} U\left(c^{i}, c^{j}\right) \pi\left(c^{i}, c^{j}\right) \operatorname{com} \partial^{2} U / \partial c^{i} \partial c^{j}<0$, os incrementos das probabilidades fora da diagonal principal da matriz $X$, geram maior bem-estar social.

\footnotetext{
${ }^{7}$ Esta transformação é realizada por observar-se que as funções de bem-estar separáveis não são compatíveis com estudos de mobilidade. Para detalhes, ver Atkinson e Bourguignon (1982).
} 
No entanto, para que isto ocorra, é necessário que a aversão social à desigualdade domine às flutuações intertemporais no consumo. Sendo assim, a matriz, apresentada no início desta subseção, tornar-se-á

$$
X^{*} \equiv\left(\begin{array}{llll}
\pi\left(c^{B}, c^{B}\right)= & 0 & \pi\left(c^{B}, c^{A}\right)= & 1 \\
\pi\left(c^{A}, c^{B}\right)= & 1 & \pi\left(c^{A}, c^{A}\right)= & 0
\end{array}\right)
$$

no ponto de ótimo. Ou seja, o $\delta$-ótimo será igual a 1 .

Vejamos este problema à luz das preferências homotéticas. Neste caso, (A.1) poderá ser representada por:

com

$$
W=\left\{\sum_{i} \sum_{j} V^{1-\epsilon} \pi\left(c^{i}, c^{j}\right)\right\}^{1 /(1-\epsilon)}
$$

$$
V=\left(\alpha_{1}\left(c^{i}\right)^{1-\rho}+\alpha_{2}\left(c^{j}\right)^{1-\rho}\right)^{1 /(1-\rho)}
$$

onde $\alpha_{i}$ representa a fração (peso) do consumo destinada a cada período, o parâmetro $\epsilon$ mede o grau de aversão social à desigualdade nos diversos períodos e $\rho$ representa a aversão às flutuações intertemporais no consumo. Como já destacado, $d W / d \delta$ será maior do que zero, se e somente se $\epsilon$ for maior do que $\rho$. Ou seja, a aversão social à desigualdade supere as flutuações intertemporais no consumo. Neste caso, o $\delta$-ótimo será igual a 1. Logo, conclui-se que a preferência pela mobilidade $(\delta \neq 0)$ induz uma situação de completa reversibilidade $(\delta=1)$.

Seguindo sua construção teórica, Atkinson e Bourguignon (1982) reinterpretam a função de bemestar social (A.2) a partir de um modelo de escolha sob incerteza (utilidade esperada), gerando:

$$
W=\left\{E_{0}\left[\alpha_{1}\left(c_{1}\right)^{1-\rho}+\alpha_{2}\left(c_{2}\right)^{1-\rho}\right]^{(1-\epsilon) /(1-\rho)}\right\}^{1 /(1-\epsilon)}
$$

Onde $E_{0}$ corresponde ao operador de esperança no tempo zero. No entanto, na sociedade representada por (A.3) não há dúvida quanto à trajetoria do consumo no período 2. Logo, conclui-se que o modelo de Atkinson-Bourguignon não é compatível com a hipótese da independência da origem. ${ }^{8}$

Por conta disso, Gottschalk e Spolaore (2002) propõem uma estrutura onde a revelação do consumo no período 1 , não implique na remoção da incerteza quanto ao consumo no período 2 . Neste caso, os agentes formularão suas expectativas quanto a $c_{2}$ a partir da observação de $c_{1}$ e da distribuição conjunta dos possíveis resultados para $c_{2}$. O ponto de partida para a formulação do modelo será a inclusão do parâmetro de aversão ao risco no segundo período $(\gamma)$. Neste caso, essa aversão pode ser vista como uma extensão dinâmica do "véu da ignorância", 9 ou seja, a incerteza é parcialmente removida no primeiro período (com o conhecimento do nível de consumo), mas mantida no período subseqüente. Sendo assim, o equivalente-certeza do consumo, no segundo período, será:

$$
\hat{c}_{2}=\left\{E_{1}\left[c_{2}^{1-\gamma}\right]\right\}^{1 / 1-\gamma}
$$

Onde $E_{1}$ corresponde a expectativa formulada com base nas informações contidas no período 1 . Substituindo (A.4) em (A.3) e considerando, por simplicidade, $\alpha_{1}=\alpha_{2}=1 / 2$, tem-se:

$$
\widehat{W}=\left\{E_{0}\left[(1 / 2) c_{1}^{1-\rho}+(1 / 2) \hat{c}_{2}^{1-\rho}\right]^{(1-\epsilon) /(1-\rho)}\right\}^{1 /(1-\epsilon)}
$$

\footnotetext{
${ }^{8}$ Note que, para $\epsilon>\rho$, o valor ótimo para $\delta$ será 1 , ou seja, uma situação onde vigore a completa reversibilidade. Neste caso, a "revelação" do consumo no período 1 , por exemplo, consumo baixo $\left(c^{B}\right)$, implicará, necessariamente, em um consumo alto $\left(c^{A}\right)$ no período 2. Logo, não haverá incerteza.

${ }^{9}$ Os argumentos filosóficos do "véu da ignorância" foram sintetizados por Rawls (1970). De acordo com esse princípio, os autores da escolha não possuem informação acerca de seu lugar na sociedade, a posição de classe ou estrato social, a fortuna ou distribuição de talentos naturais ou capacidades, etc. Neste sentido, há uma conexão direta com os argumentos econômicos da escolha sob incerteza. Harsanyi (1955) estabeleceu esse link.
} 
Caso $\epsilon=\rho=\gamma$, (A.5) será uma função aditiva separável de von Neumann e Morgenstern (1944) vN-M. Caso os parâmetros difiram, essa função será compatível com uma classe mais ampla de preferências, como a contida na axiomatização de Kreps e Porteus (1978). Nessa abordagem, observa-se:

a) o abandono do axioma das loterias compostas;

b) a manutenção dos demais axiomas de vN-M e;

c) consistência temporal dos planos ótimos de escolha.

A compatibilidade desta estrutura com a hipótese da independência da origem é estabelecida pelas proposições de Gottschalk e Spolaore (2002) reproduzidas a seguir:

Proposição 1: A independência poderá ser avaliada se e somente se $\max \{\epsilon, \rho\}>\gamma$ e $\min \{\epsilon, \rho\} \geq \gamma$. Assim, a independência temporal poderá ser avaliada se $\epsilon \geq \gamma$ e $\rho \geq \gamma$, sendo, pelo menos, uma das igualdades estrita.

Proposição 2: Se as condições da Proposição 1 são satisfeitas, o valor de $\delta$ que maximiza o bem-estar em (2.5) é maior/igual/menor do que $1 / 2$ se $\epsilon$ for maior/igual/menor do que $\rho$.

Note que as proposições garantem a avaliação da reversibilidade e da independência da origem, tanto conjuntamente (independência temporal), quanto separadamente. Em resumo, os resultados indicam que é possível estabelecer a relação entre a mobilidade de renda e o bem-estar econômico, desde que se considere os seguintes parâmetros: a aversão à desigualdade nos múltiplos períodos $(\epsilon)$; a aversão às flutuações intertemporais $(\rho)$ e; a aversão ao risco futuro $(\gamma)$. Note que a reversibilidade reduz a desigualdade nos múltiplos períodos, mas aumenta as flutuações intertemporais. Logo, os indivíduos preferirão a reversibilidade se a aversão à desigualdade dominar a aversão às flutuações intertemporais $(\epsilon>\rho)$.

A independência da origem reduz a desigualdade nos múltiplos períodos, mas aumenta o risco relativo ao consumo futuro. Sendo assim, os indivíduos preferirão a independência da origem caso as aversões à desigualdade e as flutuações intertemporais dominarem a aversão ao risco futuro ( $\epsilon$ e $\rho$ não são menores do que $\gamma$ e pelo menos um deles é maior). A avaliação dessas situações será considerada na seção seguinte.

\section{A.2. Índice de Atkinson estendido}

Atkinson (1970) propõe uma medida de bem-estar baseada no nível de renda que vigoraria caso a distribuição fosse igualitária $\left(y_{E D I}\right)$, ou seja, o nível de renda per capita de uma sociedade onde os rendimentos fossem distribuídos igualmente entre os indivíduos, de modo a gerar nível de bem-estar equivalente ao observado na distribuição atual. Formalmente, tem-se:

$$
u\left(y_{E D I}\right) \int_{0}^{\bar{y}} f(y) d y=\int_{0}^{\bar{y}} u(y) f(y) d y
$$

Onde $\bar{y}$ é a renda mais alta observada na distribuição; $f(y)$ é a densidade da renda e; $u(y)$ uma função de utilidade individual de Bernoulli. Esta medida é inavariante às transformações lineares. Sendo assim, o índice de Atkinson será:

$$
A=1-\frac{y_{E D I}}{\mu}
$$

Onde $\mu$ representa a média empírica da renda. 
Considerando um parâmetro de aversão à desigualdade $\epsilon \geq 0$, a equação (A.6) tornar-se-á:

$$
A= \begin{cases}1-\left(\frac{1}{n} \sum_{i=1}^{n}\left(\frac{y_{i}}{\mu}\right)^{1-\epsilon}\right)^{1 / 1-\epsilon}, & \text { se } \epsilon \neq 1 \\ 1-\prod_{i=1}^{n}\left(\frac{y_{i}}{\mu}\right)^{1 / n}, & \text { se } \epsilon=1\end{cases}
$$

Ou de uma forma equivalente:

$$
A=1-\frac{W_{0}}{\mu}
$$

Sendo $W_{0}$ uma função de bem-estar social. O índice é crescente em $\epsilon$. Neste caso, quanto maior o valor de $\epsilon$, maior a importância atribuída à renda dos mais pobres. Se $\epsilon=0$, então a função de utilidade será linear e a distribuição de renda não afetará o nível de bem-estar $(A=0)$. Em suma, o índice de Atkinson mensura a perda de bem-estar oriunda da desigualdade de renda. Por exemplo, caso $A=0,1$, a sociedade poderia obter o mesmo nível de bem-estar social com $90 \%$ da renda sendo distribuída igualmente. Neste caso, a sociedade encontraria-se em um padrão elevado de bem-estar. Nesta subseção, será apresentada uma extensão para o índice de Atkinson. Serão promovidas alterações no cálculo das funções de bem-estar, de modo a incorporar os conceitos de mobilidade de renda.

Tomando a sociedade estática como o ponto de partida, considere:

a) $W_{s}$ como nível de bem-estar e;

b) que o nível de consumo do indivíduo $i$ no primeiro período $\left(c_{1}^{i}\right)$ será preservado no segundo $\left(c_{12}^{i}\right)$, assim:

$$
W_{s}=\left\{E_{0}\left[(1 / 2)\left(c_{1}^{i}\right)^{1-\rho}+(1 / 2)\left(c_{12}^{i}\right)^{1-\rho}\right]^{(1-\epsilon) /(1-\rho)}\right\}^{1 /(1-\epsilon)}
$$

Por definição, a sociedade estática não possui independência temporal. Uma vez que os indivíduos são aversos à desigualdade $(\epsilon \geq 0)$ e/ou às flutuações intertemporais $(\rho \geq 0)$, eles prefeririam uma situação onde, em cada período, o consumo fosse determinado por:

$$
\tilde{c} \equiv E_{0} \frac{c_{1}+c_{2}}{2}
$$

Neste caso, a sociedade estática apresentaria um $W_{s} \leq \tilde{c}$. A diferença entre $\tilde{c}$ e $W_{s}$ mensura o quanto a sociedade pode ganhar se a desigualdade no consumo (entre os indivíduos e ao longo dos períodos) for eliminada. Atkinson (1970) considera este problema à luz da seguinte pergunta: qual a fração de $\tilde{c}$ que a sociedade está disposta a sacrificar de modo a torná-la mais igualitária? Surge então o índice capaz de responder a essa questão:

$$
A_{s}=1-\frac{W_{s}}{\tilde{c}}
$$

Note que $A_{s}$ mensura o bem-estar "perdido" por conta da desigualdade. Considere agora a ótica da reversibilidade. Neste caso, $W_{r}$ denotará o bem-estar, onde, não há dúvida sobre o nível de consumo no período $2\left(E_{1}\left[c_{2}\right]=c_{2}\right)$. Assim:

$$
W_{r}=\left\{E_{0}\left[(1 / 2)\left(c_{1}\right)^{1-\rho}+(1 / 2)\left(c_{2}\right)^{1-\rho}\right]^{(1-\epsilon) /(1-\rho)}\right\}^{1 /(1-\epsilon)}
$$

Dessa forma, (A.7) tornar-se-á:

$$
A_{r}=1-\frac{W_{r}}{\tilde{c}}
$$

De forma análoga, $A_{r}$ mensura a fração de consumo $\tilde{c}$ que os indivíduos estão dispostos a sacrificar em favor da igualdade. Se a reversibilidade causa um maior bem-estar $(\epsilon>\rho)$ e $c_{2}^{i} \neq c_{12}^{i}$ para algum $i$, 
então $W_{r}>W_{s}$, que por sua vez implica em $A_{r}<A_{s}$. Sendo assim, a diferença $A_{s}-A_{r}$ mensurará a redução na fração do consumo que a sociedade terá de sacrificar de modo a eliminar a desigualdade. Note que, neste caso, a reversibilidade diminuiria o "sacrifício" de consumo requerido pela aversão à desigualdade.

Por fim, considere a independência da origem representada pela equação (A.5). Nesta situação, o índice de Atkinson será representado por:

$$
A_{0}=1-\frac{\widehat{W}}{\tilde{c}}
$$

A comparação de $A_{r}$ e $A_{0}$ fornecerá o impacto no bem-estar gerado pela "independência da origem". Por conseguinte, o impacto total sobre o nível de bem-estar social será: ${ }^{10}$

$$
A_{s}-A_{0} \equiv\left(A_{s}-A_{r}\right)-\left(A_{r}-A_{0}\right)
$$

Este resultado estabelece uma importante conexão entre mobilidade, em seu conceito mais amplo, e bem-estar econômico.

\footnotetext{
${ }^{10}$ Este indicador mede a perda de bem-estar. Entretanto, se ele for invertido $A_{0}-A_{s} \equiv\left(A_{r}-A_{s}\right)-\left(A_{0}-A_{r}\right)$, teremos o ganho de bem-estar associado à mobilidade.
} 